\title{
Germination rate and seedling vigour of tall fescue as affected by endophyte status and seed density
}

\author{
H.T. OWENS ${ }^{1}$, C.H. FINNESETH ${ }^{2}$, T.M. TILLERY ${ }^{2}$ and T.D. PHILLIPS \\ ${ }^{1}$ Department of Plant and Soil Sciences, ${ }^{2}$ Regulatory Services, Seed Testing Laboratory, University of Kentucky \\ tphillip@email.uky.edu
}

\begin{abstract}
Forage grass seed quality is influenced by plant genetics as well as seed density, storage conditions, age of seed, and endophyte infection status. We used eight seed lots of tall fescue (three cultivars, with natural endophytes, endophyte-free, or novel endophyte) to test the effect of seed density on germination and seedling vigour. Seed lots were separated into nine density fractions using a cylinder air column. Thousand seed weight values were determined. Three runs of four replications of plots consisting of 25 seeds of each entry were seeded in greenhouse experiments to determine seedling mass at 8 weeks after planting. Early germination counts as well as laboratory germination tests indicated significant differences among weight classes in speed of germination as well as seedling vigour. The lightest fraction of seed clearly contained inferior seed, but higher density seed lots tended to have better germination and seedling vigour than lower density ones.
\end{abstract}

Keywords: seed density, seedling vigour, germination, tall fescue, endophyte

\section{Introduction}

Tall fescue [Schedonorus phoenix (Scop.) Holub] is the predominant perennial cool season forage and turfgrass species in the transition zone of the U.S. (Buckner et al. 1979). Adverse effects on animals grazing tall fescue have been noted for many years and are attributed to alkaloids produced by tall fescue infected with its endophyte [Neotyphodium coenophialum (Morgan-Jones \& Gams) Glenn, Bacon, \& Hanlin] (Stuedemann \& Hoveland 1988). Removal of this seed-transmitted endophyte eliminates the severe detrimental effects on animals grazing the grass or consuming hay. However, agronomic performance of endophyte-free tall fescue cultivars has been less consistent than that of infected cultivars (Bacon \& Siegel 1988). New, novel endophytes without harmful effects to grazing livestock are now available in different cultivars.
Several factors contribute to the difficulties encountered in establishment and maintenance of endophyte-free (or any other) tall fescue pastures. Among these factors, low seedling vigour is partially responsible for slow establishment (Clay 1987).

Seedling vigour has been studied in grass species using numerous experimental methodologies (McKell 1972). Some of the characteristics that have been measured include seed size and mass, speed of germination, germination force, root and shoot growth rates, and time to first tiller appearance. Recent research suggests that seed density can have a large effect on germination rate in grasses (Larsen \& Andreasen 2004).

Our objectives in these experiments were to compare seedling vigour in different tall fescue seed lots (different varieties, different endophyte status) grown in the same year and location, and separated into different seed densities.

\section{Materials and Methods}

Three tall fescue cultivars with different endophyte status were used in these experiments. All seed lots were produced in 2005 at the University of Kentucky Spindletop Agronomy Research Farm. All seed lots were conditioned to commercial standards. The following eight seed lots were used: EFKY31, EIKY31, EFKYFA9301, KYFA9301/AR542, KYFA9301/AR584, EFKYFA9821, KYFA9821/AR542, and KYFA9821/AR584. Each seed lot was divided into eight weight classes (designated LLL, LLH, LHL, LHH, HLL, HLH, HHL, and HHH) by three equal separations using an air column blower (South Dakota Seed Cleaner, Seedburo, Chicago, Illinois). Each seed lot also had an 'original' sample $(\mathrm{O})$ reserved as an entry, in addition to the eight weight classes.

Standard seed laboratory germination tests (ISTA 1999) were conducted by the Seed Testing Laboratory of Regulatory Services at the University of Kentucky.

For the greenhouse seedling vigour measurements, plots consisted of 25 seeds planted in a randomised complete block design experiment with four replications and three runs.

Table 1 Analysis of variance statistics for traits measured in tall fescue seed lots separated by density.

\begin{tabular}{lccccc}
\hline Source of variation & $\begin{array}{c}\text { Final lab } \\
\text { germination }\end{array}$ & $\begin{array}{c}\text { Greenhouse } \\
\text { germination }\end{array}$ & $\begin{array}{c}\text { Seedling dry } \\
\text { weight }\end{array}$ & $\begin{array}{c}\text { Seedling dry } \\
\text { wt/plant }\end{array}$ & Tiller number \\
\hline Rep(run) & $* *$ & $* *$ & $* *$ & $* *$ & $* *$ \\
Cultivar & $* *$ & $* *$ & $* *$ & $* *$ \\
Weight class & $* *$ & $* *$ & NS & NS & $*$ \\
CV ${ }^{*}$ Wt class & $* *$ & $* *$ & 0.49 & 0.47 & 0.59 \\
$R^{2}$ & 0.95 & 0.76 & 22.98 & 24.39 & 15.60 \\
C.V. & 3.54 & 7.92 & 0.215 & 9 & 1.149 \\
LSD 5\% (cultivar) & 1.51 & 1.90 & 0.180 & 6 & 1.354 \\
LSD 5\% wt. class) & 1.60 & 2.10 & $* *$
\end{tabular}

${ }^{*},{ }^{\star *}$, NS indicates statistical significance a the $5 \%, 1 \%$ or not sigificant, respectively 
Table 2 Thousand seed weight $(\mathrm{g})$ of tall fescue seed lots of three cultivars after separation by an air column blower.

\begin{tabular}{lccccccccc}
\hline Weight & \multicolumn{2}{c}{--- KY31 --- } & \multicolumn{2}{c}{----- KYFA9301 ----- } & \multicolumn{2}{c}{----- KYFA9821 ----- } & Mean \\
class & EF & El & EF & AR542 & AR584 & EF & AR542 & AR584 & \\
\hline O & 2.087 & 1.944 & 2.617 & 2.234 & 2.299 & 2.468 & 2.383 & 2.369 & 2.300 \\
LLL & 1.123 & 0.966 & 1.512 & 1.163 & 1.417 & 1.681 & 1.388 & 1.594 & 1.356 \\
LLH & 1.938 & 1.781 & 2.369 & 2.062 & 2.057 & 2.214 & 2.099 & 2.131 & 2.081 \\
LHL & 2.109 & 1.882 & 2.413 & 2.080 & 2.236 & 2.444 & 2.338 & 2.213 & 2.214 \\
HLL & 2.241 & 2.046 & 2.669 & 2.188 & 2.374 & 2.586 & 2.425 & 2.428 & 2.370 \\
LHH & 2.410 & 2.172 & 2.785 & 2.505 & 2.520 & 2.831 & 2.668 & 2.523 & 2.552 \\
HLH & 2.632 & 2.339 & 2.953 & 2.539 & 2.602 & 2.936 & 2.776 & 2.692 & 2.684 \\
HHL & 2.605 & 2.374 & 3.045 & 2.511 & 2.718 & 2.943 & 2.958 & 2.807 & 2.745 \\
HHH & 2.916 & 2.661 & 3.330 & 2.896 & 2.940 & 3.278 & 3.198 & 3.031 & 3.031 \\
Mean & 2.229 & 2.018 & 2.633 & 2.242 & 2.351 & 2.598 & 2.470 & 2.421 & 2.356 \\
\hline
\end{tabular}

Table 3 Final germination (\%) from four replications of laboratory germination tests.

\begin{tabular}{llcccccccc}
\hline Weight & \multicolumn{2}{l}{--- KY31 } & --- & \multicolumn{2}{c}{---- KYFA9301 ----- } & \multicolumn{2}{c}{----- KYFA9821 ----- } & Mean \\
class & EF & El & EF & AR542 & AR584 & EF & AR542 & AR584 & \\
\hline O & 88 & 77 & 87 & 84 & 87 & 89 & 89 & 91 & 86.5 \\
LLL & 49 & 46 & 61 & 49 & 67 & 67 & 59 & 63 & 57.6 \\
LLH & 96 & 100 & 97 & 93 & 95 & 96 & 96 & 96 & 96.1 \\
LHL & 97 & 98 & 98 & 95 & 96 & 97 & 95 & 96 & 96.5 \\
HLL & 99 & 96 & 98 & 97 & 96 & 98 & 96 & 97 & 97.1 \\
LHH & 98 & 100 & 98 & 97 & 97 & 98 & 99 & 96 & 97.9 \\
HLH & 99 & 99 & 99 & 97 & 97 & 99 & 100 & 98 & 98.5 \\
HHL & 97 & 100 & 99 & 98 & 98 & 98 & 98 & 99 & 98.4 \\
HHH & 99 & 98 & 99 & 99 & 98 & 98 & 98 & 99 & 98.5 \\
Mean & 91.3 & 90.4 & 92.9 & 89.9 & 92.3 & 93.3 & 92.2 & 92.8 & 91.9 \\
\hline
\end{tabular}

Table 4 Final germination (\%) of seed lots in a greenhouse study averaged over three runs of four replications.

\begin{tabular}{lccccccccc}
\hline $\begin{array}{l}\text { Weight } \\
\text { class }\end{array}$ & \multicolumn{2}{c}{ KY31 } & \multicolumn{3}{c}{---- KYFA9301---- } & \multicolumn{2}{c}{---- KYFA9821---- } & Mean \\
\hline O & 83.3 & 82.3 & 89.3 & 83.3 & 84.7 & 87.0 & 83.7 & 87.7 & 85.2 \\
LLL & 50.3 & 37.0 & 61.0 & 51.0 & 64.3 & 67.7 & 60.7 & 70.0 & 57.8 \\
LLH & 88.7 & 86.0 & 90.3 & 87.3 & 88.3 & 88.7 & 87.3 & 84.3 & 87.6 \\
LHL & 91.0 & 88.0 & 91.3 & 88.0 & 89.0 & 92.0 & 93.0 & 90.7 & 90.4 \\
HLL & 91.0 & 87.0 & 92.7 & 89.3 & 91.7 & 91.0 & 92.0 & 91.3 & 90.8 \\
LHH & 93.7 & 93.3 & 87.7 & 94.0 & 91.3 & 93.3 & 90.3 & 91.0 & 91.8 \\
HLH & 93.0 & 91.7 & 95.0 & 91.7 & 91.7 & 92.0 & 90.7 & 93.7 & 92.4 \\
HHL & 92.7 & 92.3 & 92.3 & 95.3 & 92.7 & 94.3 & 93.7 & 93.7 & 93.4 \\
HHH & 91.0 & 92.3 & 93.3 & 95.3 & 93.7 & 95.7 & 95.7 & 94.7 & 94.0 \\
Mean & 86.1 & 83.3 & 88.1 & 86.1 & 87.5 & 89.1 & 87.5 & 88.6 & 87.0 \\
\hline
\end{tabular}


Table 5 Seedling dry weight $(\mathrm{g})$ for seed lots averaged over three runs of four replications in a greenhouse study.

\begin{tabular}{lccccccccc}
\hline \multirow{2}{*}{$\begin{array}{l}\text { Weight } \\
\text { class }\end{array}$} & EF & EI & EF & AR542 & AR584 & EF & AR542 & AR584 & \\
\hline O & 2.198 & 2.903 & 2.816 & 3.173 & 3.094 & 2.796 & 2.625 & 3.195 & 2.850 \\
LLL & 2.035 & 1.606 & 2.524 & 1.878 & 2.621 & 2.506 & 2.168 & 2.537 & 2.234 \\
LLH & 2.873 & 2.781 & 3.119 & 2.877 & 2.531 & 2.697 & 2.808 & 3.224 & 2.864 \\
LHL & 2.673 & 2.964 & 2.668 & 2.911 & 3.006 & 2.980 & 2.874 & 2.977 & 2.882 \\
HLL & 2.561 & 2.971 & 2.716 & 2.806 & 2.951 & 2.828 & 2.981 & 2.828 & 2.830 \\
LHH & 2.716 & 2.712 & 3.068 & 2.907 & 2.964 & 2.956 & 3.073 & 2.751 & 2.893 \\
HLH & 2.645 & 3.156 & 3.234 & 3.035 & 3.348 & 3.119 & 2.856 & 3.201 & 3.074 \\
HHL & 2.955 & 3.189 & 2.885 & 2.955 & 2.783 & 3.336 & 3.355 & 3.444 & 3.113 \\
HHH & 2.838 & 3.194 & 3.516 & 3.000 & 3.342 & 3.337 & 2.895 & 3.418 & 3.193 \\
Mean & 2.610 & 2.831 & 2.950 & 2.838 & 2.960 & 2.951 & 2.848 & 3.064 & 2.881 \\
\hline
\end{tabular}

Table 6 Tiller number per plot (from 25 seeds) for seed lots averaged over three runs of four replications.

\begin{tabular}{lccccccccc}
\hline $\begin{array}{l}\text { Weight } \\
\text { class }\end{array}$ & EF & EI & EF & AR542 & AR584 & EF & AR542 & AR584 & \\
\hline O & 25.5 & 28.4 & 27.9 & 27.7 & 31.1 & 31.6 & 29.1 & 32.2 & 29.2 \\
LLL & 19.2 & 15.3 & 24.2 & 19.3 & 23.5 & 25.4 & 23.4 & 25.8 & 22.0 \\
LLH & 29.8 & 27.3 & 30.4 & 28.4 & 27.2 & 28.5 & 29.7 & 30.5 & 29.0 \\
LHL & 29.8 & 28.4 & 28.0 & 29.0 & 30.8 & 29.2 & 30.2 & 30.5 & 29.5 \\
HLL & 28.9 & 29.0 & 29.4 & 28.9 & 28.2 & 30.7 & 30.3 & 31.8 & 29.7 \\
LHH & 29.8 & 28.8 & 32.2 & 27.8 & 31.0 & 28.8 & 31.1 & 29.2 & 29.8 \\
HLH & 30.2 & 30.3 & 31.4 & 29.4 & 31.9 & 33.2 & 29.2 & 32.1 & 31.0 \\
HHL & 29.6 & 27.9 & 28.8 & 31.1 & 30.2 & 34.4 & 31.3 & 35.5 & 31.1 \\
HHH & 31.4 & 31.2 & 31.5 & 32.1 & 33.1 & 34.1 & 31.9 & 34.7 & 32.5 \\
Mean & 28.2 & 27.4 & 29.3 & 28.2 & 29.7 & 30.7 & 29.6 & 31.4 & 29.3 \\
\hline
\end{tabular}

Table 7 Seedling dry weight/plant $(\mathrm{mg})$ for seed lots averaged over three runs of four replications in a greenhouse study.

\begin{tabular}{lccccccccc}
\hline $\begin{array}{l}\text { Weight } \\
\text { class }\end{array}$ & EF & EI & EF & AR542 & AR584 & EF & AR542 & AR584 & \\
\hline O & 106 & 142 & 127 & 154 & 149 & 129 & 126 & 147 & 135 \\
LLL & 162 & 185 & 171 & 150 & 164 & 152 & 142 & 150 & 160 \\
LLH & 131 & 129 & 137 & 132 & 114 & 123 & 131 & 156 & 132 \\
LHL & 117 & 134 & 117 & 134 & 136 & 129 & 124 & 130 & 128 \\
HLL & 114 & 137 & 117 & 127 & 131 & 124 & 131 & 125 & 126 \\
LHH & 117 & 117 & 141 & 124 & 130 & 127 & 139 & 125 & 128 \\
HLH & 114 & 140 & 137 & 132 & 145 & 136 & 128 & 138 & 134 \\
HHL & 126 & 139 & 125 & 123 & 121 & 143 & 143 & 149 & 134 \\
HHH & 125 & 139 & 152 & 126 & 143 & 140 & 121 & 145 & 136 \\
Mean & 124 & 140 & 136 & 134 & 137 & 134 & 132 & 141 & 135 \\
\hline
\end{tabular}


Containers were $8 \mathrm{~cm} \times 12 \mathrm{~cm} \times 6 \mathrm{~cm}$ plastic trays with a peatbased growing medium. Trays were placed in a greenhouse during the summer 2006, with natural sunlight. Seedling numbers were counted for 3 weeks, and 55-60 days after planting, tiller number and above ground seedling mass (after oven drying) were recorded for each plot. Statistical analyses of data were made using the general linear model procedure of SAS (SAS 1988).

\section{Results and Discussion}

All traits showed highly significant differences among cultivars and among weight classes (Table 1). Thousand seed weights (Table 2) confirmed differences in initial seed weights among seed lots. For all three cultivars, the endophyte-free seed lots had higher germination percent than infected seed lots (Tables 3 \& 4), with means for EFKY31 and EFKYFA9301 being statistically significantly higher than the means for EIKY31 and KYFA9301/AR542, respectively; and strain AR584 seed lots had superior germination (in the greenhouse) to seed lots containing strain AR542. The LLL (lightest weight class) had much lower germination than other weight classes (mainly due to $\sim 50 \%$ empty seed, data not shown). Seedling mass was greater in infected seed lots than endophyte-free (Table 5). The three heaviest weight classes produced the greatest seedling mass of all weight classes. EFKY31 produced more tillers than EIKY31, and strain AR584-containing seed lots had more tillers than EF or AR542 seed lots of KYFA9301 or KYFA9821 (Table 6). Again, the three heaviest weight classes had superior tiller numbers, with LLL having the fewest. To account for some of the confounding effects of germination on other traits, weight per plant was analysed (Table 7). EIKY31 and KYFA9821/AR584 had the heaviest per plant weight, as did the LLL weight class (with fewer plants germinating, each plant had more space to grow). Tillers per plant (data not shown) were highest in the LLL seed lots for the same reason, and KYFA9821/AR584 produced more tillers than other combinations.

Seedling vigour can be improved by removing the lightest seed from the seed lots. These results provide evidence that novel endophytes confer superior seedling vigour, often better than the common, toxic endophyte in KY31.

\section{REFERENCES}

Bacon, C.W.; Siegel, M.R. 1988. Endophyte parasitism of tall fescue. Journal of Production Agriculture 1: 45-55.
Badoux, S. 1977. Breeding tall fescue (Festuca arundinacea Schreb.) and cocksfoot (Dactylis glomerata L.) for seedling establishment under conditions of competition. pp. 443-448. In: Proceedings of the XIII International Grasslands Congress.

Buckner, R.C.; Powell, J. B.; Frakes, R.V. 1979. Historical development. pp. 1- 8. In: Tall Fescue. Eds. Buckner; R.C.; Bush, L.P. Agronomy Monograph 20.

Clay, K. 1987. Effects of fungal endophytes on the seed and seedling biology of Lolium perenne and Festuca arundinacea. Oecologia 73: 358-362.

Easton, H.S.; Pennell C.G. 1993. Breeding tall fescue for establishment vigour. Proceedings of the New Zealand Grassland Association 55: 151-154.

Glenn, A.E.; Bacon, C.W.; Price, R.; Hanlin, R.T. 1996. Molecular phylogeny of Acremonium and its taxonomic implications. Mycologia 88(3): 369-383.

International Seed Testing Association. 1987. Handbook of vigour test methods. International Seed Testing Association, Zurich, Switzerland.

International Seed Testing Association. 1999. International rules for seed testing: Rules 1999. Seed Science and Technology 27(suppl.):1-333.

Kneebone, W.R. 1972. Breeding for seedling vigor. pp. 90-100. In: The Biology and Utilization of Grasses. Eds. Youngner, V.B.; McKell, C.M., Academic Press, New York.

Larsen, S.U.; Andreasen, C. 2004. Light and heavy turfgrass seeds differ in germination percentage and mean germination thermal time. Crop Science 44: 1710-1720.

Lewis, E. J.; Garcia, J.A. 1979. The effect of seed mass and coleoptile tiller development on seedling vigour in tall fescue, Festuca arundinacea. Euphytica 28: 393-402.

McKell, C.M. 1972. Seedling vigor and seedling establishment. pp. 74-89. In: The Biology and Utilization of Grasses. Eds. Youngner, V.B.; McKell, C.M., Academic Press, New York.

Robinson, L.R.; Thomas, H.L. 1963. Combining ability for seedling vigour in Bromus inermis. Crop Science 3: 358-359.

SAS Institute Inc., 1988. SAS/STAT users guide, release 6.03 edition. Cary, NC: SAS Institute Inc.

Stuedemann, J.A.; Hoveland, C.S. 1988. Fescue endophyte: history and impact on animal agriculture. Journal of Production Agriculture 1: 39-44. 